\title{
Preliminary Identification of Human Leukocyte Antigen (HLA) CLASS II DRB1 Allelic Variants in Selected Filipino Cancer Patient Samples
}

\author{
Jemicah Tristian P. Cobarrubias, ${ }^{1}$ Ciara Christianne Y. Lim, ${ }^{1}$ Ma. Teresa A. Barzaga ${ }^{2}$ and Francisco M. Heralde III ${ }^{2,3}$ \\ ${ }^{1}$ Department of Physical Sciences and Mathematics, College of Arts and Sciences, University of the Philippines Manila \\ ${ }^{2}$ Molecular Diagnostics and Cellular Therapeutics Laboratory Pathology Department, Lung Center of the Philippines \\ ${ }^{3}$ Department of Biochemistry and Molecular Biology, College of Medicine, University of the Philippines Manila
}

\begin{abstract}
Objective. The Human Leukocyte Antigen (HLA) Class II is the major histocompatibility complex surface glycoproteins of humans responsible for presenting exogenous antigenic peptides which help direct specificity of immune response. In immune-cell therapy, the HLA allelic variants are of particular importance as they determine the successful activation of target cells that results to a desired therapeutic response. However, HLA Class II exhibits high polymorphism and has variable distribution in population, constituting these so-called allelic variants. Specifically, the HLA Class II DRB1 is considered the predominant locus among Filipinos. This research aimed to identify the presence of HLA Class II DRB1 allelic variants in the stem cell samples of ten (10) Filipino cancer patients by reverse transcription polymerase chain reaction (RT-PCR) amplification.
\end{abstract}

Method. This study employed a PCR-based HLA Class II typing to identify the HLA Class II DRB1 allelic variant in Filipino cancer patients. Design of forward and reverse primers for HLA Class II DRB1, optimization of PCR conditions for amplifying HLA Class II DRB1, and identification of HLA Class II DRB1 allelic variants from samples by sequencing and database comparison were conducted.

Results. PCR optimization showed that optimum annealing temperature for HLA DRB1 was $58.8^{\circ} \mathrm{C}$ with 1 mM $\mathrm{MgCl}_{2}$. PCR amplification of HLA DRB1 from ten anonymized cancer patient samples and DNA sequencing revealed that Patients 1, 2, 5, 8, 9, and 10 harbor HLA DRB1 allelic variants, particularly, the HLA DRB1*04:06:01, HLA DRB1*12:01:01, HLA DRB1*0813, HLA DRB1*04:05:01, HLA DRB1*09:01:02, and HLA DRB1*16:02:01, allelic variants, respectively.

Conclusion. Using the designed primers and optimized RT-PCR protocol, HLA information derived from six out of ten patient samples can be used for further applications in developing personalized or generic antigenic peptides such as dendritic cell cancer vaccine.

Key Words: HLA Class II DRB1, allelic variants, PCR-Based HLA Typing, cancer immune-cell therapy

\section{INTRODUCTION}

Corresponding author: Jemicah Tristian P. Cobarrubias Department of Physical Sciences and Mathematics College of Arts and Sciences

University of the Philippines Manila

Padre Faura St., Ermita, Manila 1000, Philippines

Email: tristian.cobarrubias@gmail.com
The Human Leukocyte Antigen (HLA) is the major histocompatibility complex (MHC) of humans that plays a major role in eliciting the adaptive immune response by activating the body's $\mathrm{T}$ cells. This, in turn, results in the activation of $\mathrm{B}$ cells, recruitment of macrophages, neutrophils, natural killer (NK) cells, cytokines, and antimicrobial peptides that attack foreign or pathogenic bodies. ${ }^{1}$ The HLA Class I present intracellular-derived peptides to $\mathrm{CD} 8+\mathrm{T}$ cells while HLA Class II present exogenous peptides to CD4+ $\mathrm{T}$ cells. ${ }^{2}$ Thus, therapeutic interventions involving extracellular antigens must be 
presented via HLA Class II molecules, which are only expressed by antigen-presenting cells. ${ }^{3}$

The classical HLA Class II genes: HLA-DP, HLADQ, and HLA-DR are highly polymorphic such that more than 300 alleles are found in each of the loci mentioned. ${ }^{4}$ Recent database information shows that there are around 3,920 HLA class II identified, which exemplify the genomic diversity of HLA. ${ }^{5}$ Investigations on the frequencies of the different HLA alleles reveal that certain variants are expressed predominantly in specific populations. In particular, a study of HLA distribution among Filipinos showed that HLA DRB1*1502 is the most commonly occurring HLA Class II allele. ${ }^{6}$ As of the time of writing, no follow up investigation has been conducted on HLA typing in Filipinos except for this study.

HLA typing by PCR is one of the most utilized molecular techniques in determining the allele present in an HLA locus. One significant clinical application for HLA typing is its use in tissue/organ transplant wherein cross-matching between immune cells of donors and antibodies of recipients are thoroughly investigated. ${ }^{7}$ HLA typing can also be applied in designing antigens for cancer immunotherapies such as dendritic cell vaccines. The downstream application to immune cell therapy may involve allogeneic dendritic cell therapy and this entails specific types of HLA alleles to be identified for developing antigenic cancer peptides against them.

Currently, identification of HLA allelic variants among cancer patients is not part of the stem cell immunotherapeutic program of the Lung Center of the Philippines. However, by knowing the type of HLA allele and the type of peptide it can present, appropriate antigenic peptide design can be done to elicit the optimum response from the dendritic cell and its subsequent activation of $\mathrm{T}$ cells. The ideal activity of HLA DR helper T cell epitope was also proven to be valuable in generating an efficient cytotoxic $T$ cell response in cancer immunotherapy. ${ }^{8}$ Further, developing a low-cost, in-house, and PCR-based HLA typing specific to a target HLA will be beneficial since commercial versions are more generic in their typing strategies, and have little use in the dendritic cell vaccination protocol due to minimal specificity. There are also no data available on the commercial typing for Filipino patients specifically, for those who availed immune cell therapy. Thus, this study aimed to identify the expression of HLA Class II DRB1 allelic variants in Filipino cancer patient cell samples as a platform for the development of effective cancer immunotherapy vaccines.

Moreover, this research aimed to develop a reverse transcription polymerase reaction (RT-PCR) amplification protocol to identify the presence of HLA Class II DRB1 allelic variants in ten (10) Filipino cancer patient cell samples. The specific objectives of this investigation were the following: 1) to design forward and reverse primers for HLA Class II DRB1, 2) to optimize PCR conditions for amplifying HLA Class II DRB1, and 3) to identify HLA
Class II DRB1 allelic variants from samples by sequencing and database comparison.

\section{MATERIALS AND METHODS}

\section{Sample Acquisition}

Ten anonymized unstimulated stem cell samples from cancer patients were obtained from the Molecular Diagnostics and Cellular Therapeutics Laboratory (MDCTL) of the Lung Center of the Philippines (LCP). These stored samples have been previously collected by physicians and members of the MDCTL team from patients under the stem cell therapeutics program of the LCP who have signed written consent forms for the use of their samples for future studies to improve the immune cell therapy of MDCTL.

\section{Total RNA Extraction}

The total RNA from the frozen samples were collected using RNeasy kit (Qiagen GmbH, Hilden, Germany) according to the manufacturer's instructions. Quantitation of total RNA was done using a QuantusFluorometer. Samples were stored at $-80^{\circ} \mathrm{C}$ until use.

\section{cDNA Synthesis}

cDNA synthesis $(10 \mu \mathrm{L})$ of the RNA samples was done using $0.5 \mu \mathrm{L}$ of $50 \mu \mathrm{M}$ Oligo(dT)20, $0.5 \mu \mathrm{L}$ of $10 \mathrm{mM}$ dNTP, $5.1 \mu \mathrm{L}$ of sterile nuclease-free water, and $0.4 \mu \mathrm{L}$ of RNA sample heated at $65^{\circ} \mathrm{C}$ for 5 minutes and consequent dropping of temperature to $4^{\circ} \mathrm{C}$ for 2 minutes. The mixture was centrifuged briefly and was added with 2 $\mu \mathrm{L}$ of $5 \mathrm{X}$ first-strand buffer, $0.5 \mu \mathrm{L}$ of $0.1 \mathrm{M}$ DTT, and 1 $\mu \mathrm{L}$ of 200 units $/ \mu \mathrm{L}$ SuperScript ${ }^{\mathrm{TM}}$ II Reverse Transcriptase (Invitrogen, California, United States). The mixture was incubated at $25^{\circ} \mathrm{C}$ for 5 minutes and at $55^{\circ} \mathrm{C}$ for 60 minutes. Finally, the reaction was inactivated by heating at $70^{\circ} \mathrm{C}$ for 15 minutes. Samples were stored at $-20^{\circ} \mathrm{C}$ until use.

\section{Primer Design for HLA DRB1}

Primers were designed against the HLA Class II DRB1*1502 ${ }^{6}$ variant to target conserved regions of the gene using the nucleotide sequence from the Immuno Polymorphism Database-ImMunoGeneTics/ Human Leukocyte Antigen (IPD-IMGT/HLA) with accession number: HLA008675 (Appendix A). The OligoAnalyzer 3.1 of Integrated DNA Technologies was used for designing the forward and reverse primers. The resulting primers were analyzed with Primer-BLAST. In the absence of cross-reactions with other non-HLA DRB sequences, the primers were ordered from Integrated DNA Technologies, Singapore.

\section{PCR Optimization}

Gradient PCR reactions were performed with the PCR reaction mix shown in Table 1 , and ran in a T100 ${ }^{\mathrm{TM}}$ Bio-Rad Thermal Cycler (USA). 
Cycling was performed with the conditions shown in Table 2. Amplifications were confirmed by running the PCR products in $1.5 \%$ agarose gels in $1 \mathrm{X}$ Tris-borateEDTA (TBE) buffer $(0.054 \mathrm{~g} / \mathrm{mL}$ Tris base, $0.028 \mathrm{~g} / \mathrm{mL}$ Boric acid, and $0.5 \mathrm{M} \mathrm{Na}_{2}$ EDTA).

\section{Reverse Transcription PCR Amplification}

PCR reactions $(10 \mu \mathrm{L})$ were performed for the ten cancer patient samples with the PCR reaction mix shown in Table 3, and ran in a T100 ${ }^{\mathrm{TM}}$ Bio-Rad Thermal Cycler (USA).

Cycling was performed with the conditions shown in Table 4. Amplifications were confirmed by running them in $1.5 \%$ agarose gels.

\section{DNA Sequencing}

Samples that were positive for amplification from the AGE run were gel-purified using GF-1 Gel DNA Recovery Kit (Vivantis, Malaysia) and $30 \mu \mathrm{L}$ of the DNA sample (at least $50 \mathrm{ng} / \mu \mathrm{L}$ ) were sent to Macrogen, Korea for sequencing. The resulting sequences were analyzed by obtaining consensus sequences for the ten cancer patient samples for the forward and reverse sequences via MEGA7. The consensus sequences were run with NCBI BLAST to identify the HLA DRB1 allelic variant obtained in each Filipino cancer patient sample.

\section{RESULTS AND DISCUSSION}

\section{Analysis of Primers for HLA DRB1}

The forward primer designed against HLA DRB1*1502 (IPD-IMGT/HLA Accession No. HLA00867) was 5'ATG GTG TGT CTG AAG CTC CCT G-3'. According to OligoAnalyzer 3.1, the forward primer has a melting temperature of $59.1^{\circ} \mathrm{C}$ and $\mathrm{GC}$ content of $54.5 \%$ which implies that it falls under the recommended characteristics of a desirable primer. In general, primers with a melting temperature in the range of 50 to $62^{\circ} \mathrm{C}$ produce more desirable results and this is important to note since identifying the value for melting temperature is the most crucial part for a PCR analysis to be successful. ${ }^{9}$

The reverse primer designed using the IPD-IMGT/ HLA nucleotide sequence HLA DRB1*1502 was 5'- TCA GCT CAG GAA TCC TGT TGG C - 3'. According to OligoAnalyzer 3.1 (Integrated DNA Technologies), the reverse primer has a melting temperature of $59.4^{\circ} \mathrm{C}$ and $\mathrm{GC}$ content of $54.5 \%$.

Using Primer Blast from NCBI, the designed primers are shown to be specific to HLA DRB1 but may be crossreactive with HLA Class II allelic variants DRB3 and DRB4 since HLA DRB allelic variants exhibit high sequence similarity with differences in a small number of nucleotides. Nevertheless, the primers can still be used because of their specificity. From Primer BLAST, the length for HLA DRB1 is predicted to be $801 \mathrm{bp}$.
Table 1. Reaction concentration and volumes for optimization of annealing temperature

\begin{tabular}{|c|c|c|}
\hline Component and Reagent & $\begin{array}{c}\text { Final } \\
\text { Concentration }\end{array}$ & $\begin{array}{l}\text { Required } \\
\text { Volume }(\mu \mathrm{L})\end{array}$ \\
\hline 5X Green GoTaq ${ }^{\circledR}$ Flexi Buffer & $1 X$ & 2.00 \\
\hline $25 \mathrm{mM} \mathrm{MgCl}_{2}$ & $2.5 \mathrm{mM}$ & 1.00 \\
\hline $10 \mathrm{mM}$ dNTP & $0.2 \mathrm{mM}$ & 0.20 \\
\hline $\begin{array}{l}5 \text { units } / \mu \mathrm{L} \text { GoTaq }{ }^{\circledR} \text { Flexi DNA } \\
\text { Polymerase }\end{array}$ & 0.125 units $/ \mu \mathrm{L}$ & 0.25 \\
\hline $10 \mu \mathrm{M}$ Forward primer & $1 \mu \mathrm{M}$ & 1.00 \\
\hline $10 \mu \mathrm{M}$ Reverse primer & $1 \mu \mathrm{M}$ & 1.00 \\
\hline cDNA template & & 0.50 \\
\hline Sterile nuclease-free water & Up to $10 \mu \mathrm{L}$ & 4.05 \\
\hline
\end{tabular}

Table 2. PCR cycling conditions for optimization of annealing temperature

\begin{tabular}{lcc}
\multicolumn{1}{c}{ Process } & Time & Temperature \\
\hline Initial denaturation & $2 \mathrm{~min}$ & $94^{\circ} \mathrm{C}$ \\
Denaturation $^{\mathrm{a}}$ & $30 \mathrm{~s}$ & $94^{\circ} \mathrm{C}$ \\
Annealing $^{\mathrm{a}}$ & $30 \mathrm{~s}$ & $55-65^{\circ} \mathrm{C}$ \\
Extension $^{\mathrm{a}}$ & $1 \mathrm{~min}$ & $72^{\circ} \mathrm{C}$ \\
Final extension $^{\circ}$ & $5 \mathrm{~min}$ & $72^{\circ} \mathrm{C}$ \\
\hline
\end{tabular}

${ }^{a}$ Denaturation, annealing, and extension were set to undergo 35 cycles

Table 3. Reaction concentration and volumes for RT PCR amplification

\begin{tabular}{|c|c|c|}
\hline Component and Reagent & $\begin{array}{c}\text { Final } \\
\text { Concentration }\end{array}$ & $\begin{array}{l}\text { Required } \\
\text { Volume }(\mu \mathrm{L})\end{array}$ \\
\hline 5X Green GoTaq ${ }^{\circledR}$ Flexi Buffer & $1 \mathrm{X}$ & 2.00 \\
\hline $25 \mathrm{mM} \mathrm{MgCl}_{2}$ & $1 \mathrm{mM}$ & 0.40 \\
\hline $10 \mathrm{mM}$ dNTP & $0.2 \mathrm{mM}$ & 0.20 \\
\hline $\begin{array}{l}5 \text { units } / \mu \mathrm{L} \text { GoTaq }{ }^{\circledR} \text { Flexi DNA } \\
\text { Polymerase }\end{array}$ & 0.125 units $/ \mu \mathrm{L}$ & 0.25 \\
\hline $10 \mu \mathrm{M}$ Forward primer & $0.2 \mu \mathrm{M}$ & 0.20 \\
\hline $10 \mu \mathrm{M}$ Reverse primer & $0.2 \mu \mathrm{M}$ & 0.20 \\
\hline cDNA template & & 0.50 \\
\hline Sterile nuclease-free water & Up to $10 \mu \mathrm{L}$ & 6.25 \\
\hline
\end{tabular}

Table 4. PCR cycling conditions for RT PCR amplification

\begin{tabular}{lcc}
\multicolumn{1}{c}{ Process } & Time & Temperature \\
\hline Initial denaturation & $2 \mathrm{~min}$ & $94^{\circ} \mathrm{C}$ \\
Denaturation $^{\mathrm{a}}$ & $30 \mathrm{~s}$ & $94^{\circ} \mathrm{C}$ \\
Annealing $^{\mathrm{a}}$ & $30 \mathrm{~s}$ & $55-65^{\circ} \mathrm{C}$ \\
Extension $^{\mathrm{a}}$ & $1 \mathrm{~min}$ & $72^{\circ} \mathrm{C}$ \\
Final extension $^{\circ}$ & $5 \mathrm{~min}$ & $72^{\circ} \mathrm{C}$ \\
\hline
\end{tabular}

${ }^{a}$ Denaturation, annealing, and extension were set to undergo 35 cycles

\section{Integrity of Purified Total RNA}

Total RNA from ten frozen unstimulated stem cell samples of Filipino cancer patients was obtained using RNeasy kit from Qiagen. The results for the quantitation of total RNA was using a QuantusFluorometer as shown in Appendix B. The gel profile of the extracted total RNA is shown in Figure 1. 
Intense and bright bands corresponding to more than $3000 \mathrm{bp}$ indicates the presence of an intact RNA. RNA of all the patient samples were successfully purified. Mammalian mRNA that is intact appears as a band ranging from 4-7 $\mathrm{kb}$ with faint $28 \mathrm{~S} \mathrm{rRNA}$ and $18 \mathrm{~S}$ rRNA bands with sizes of around $2000 \mathrm{bp}$ and $750 \mathrm{bp}$, respectively ${ }^{10}$, which explains the two smaller bands observed at Lanes 2 and 5 (Figure 1).

The concentration of the RNA isolated from the frozen unstimulated stem cell samples ranged from $18-105 \mathrm{ng} / \mu \mathrm{L}$ making them suitable templates for $\mathrm{cDNA}$ synthesis. ${ }^{11}$

\section{Integrity of cDNA Synthesized}

cDNA was constructed from the mRNA of the ten (10) frozen unstimulated stem cell samples of Filipino cancer patients using SuperScript ${ }^{\mathrm{TM}}$ II Reverse Transcriptase from (Invitrogen). The gel profile of the cDNA synthesis is shown in Figure 2.

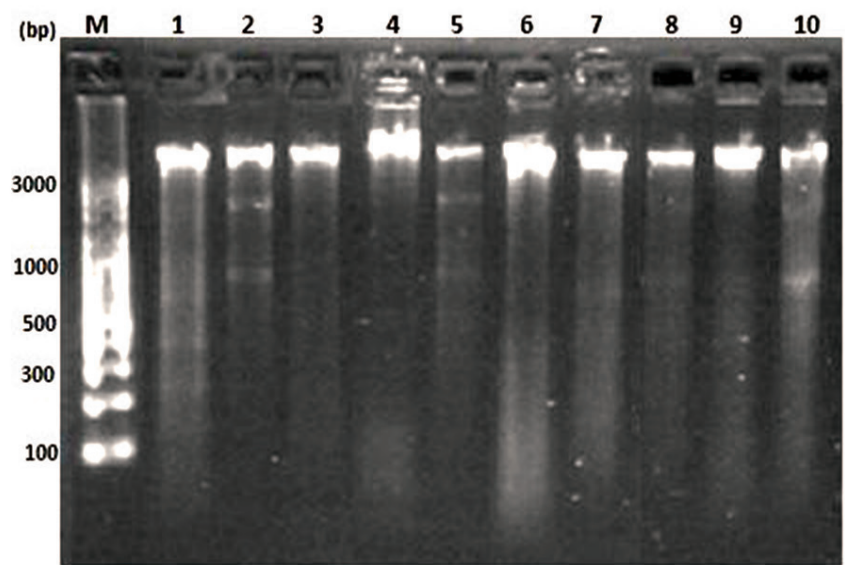

Figure 1. AGE profile of total RNA collected from unstimulated stem cell samples (Lanes 1-10) using Qiagen RNeasy Kit ran through $1.5 \%$ agarose gels in $1 \mathrm{X}$ TBE with VC 100bp Plus DNA Ladder as the MW Marker (M).

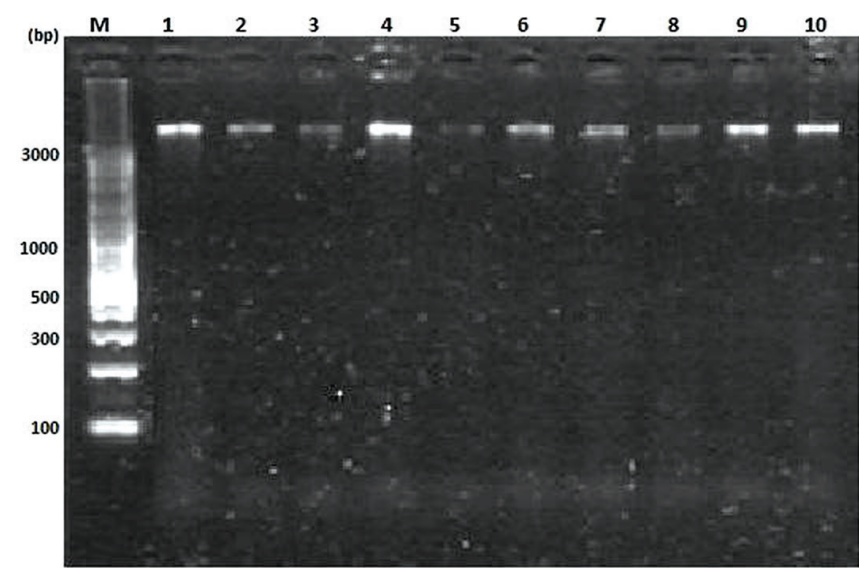

Figure 2. AGE profile of cDNA generated from RNA samples (Lanes 1-10) run through $1.5 \%$ agarose gels in $1 \mathrm{X}$ TBE with VC 100bp Plus DNA Ladder as the MW Marker (M).
The presence of prominent bands at greater than 3 $\mathrm{kb}$ indicates a successful synthesis of cDNA which can be used as template for PCR reactions to identify the presence of HLA Class II DRB1 allelic variants in Filipino cancer patient samples.

\section{PCR Optimization of Annealing Temperature and $\mathrm{Mg}^{2+}$ Concentration}

The proper annealing of primers in a PCR reaction is dependent on multiple factors such as buffer composition, concentration of primers, concentration of $\mathrm{Mg}^{2+}$, and the annealing temperature. ${ }^{12}$ Of these, the optimum annealing temperature and $\mathrm{Mg}^{2+}$ concentration are the most commonly altered parameters to increase PCR specificity. Consequently, there were the parameters altered for the amplification of the HLA Class II DRB1. Patient 1 and Patient 5 cDNA were subjected to gradient PCR with varying annealing temperatures at 55 to $65^{\circ} \mathrm{C}$ (Figure 3 ).

Lanes 1-8 and lanes 9-16 correspond to Patient 1 and Patient 5, respectively. From the gel profile, it can be seen that lanes 4 and 12 exhibited bands that are the most distinct and separated. This corresponds to an annealing temperature of $58.8^{\circ} \mathrm{C}$ for both patient samples. Lanes 1-3 and 9-11 correspond to annealing temperatures of $55^{\circ} \mathrm{C}$, $55.7^{\circ} \mathrm{C}$, and $56.9^{\circ} \mathrm{C}$, respectively. Bands can be observed from these lanes but are not distinguishable from each other as indicated by the large smear present in lanes 1-3 and 9-11. In a study conducted by Ruiz-Villalba et al. (2017) for the optimization of annealing temperature for amplification of DNA in vitro, results show that unwanted PCR products were formed at annealing temperatures that were set below and above the calculated annealing temperature. ${ }^{13}$ This can

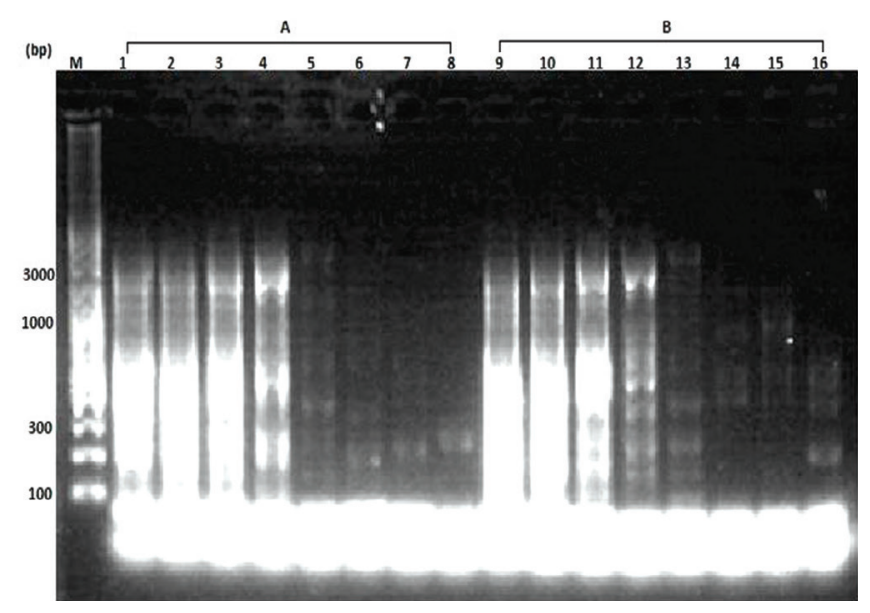

Figure 3. AGE profile of gradient PCR of (A) Patient 1 and (B) Patient 5 with varying annealing temperatures. Lanes $1,9-55^{\circ} \mathrm{C}$; Lanes $2,10-55.7^{\circ} \mathrm{C}$; Lanes 3,11 - 56.9 ${ }^{\circ} \mathrm{C}$; Lanes $4,12-58.8^{\circ} \mathrm{C}$; Lanes $5,13-61.1^{\circ} \mathrm{C}$; Lanes $6,14-63^{\circ} \mathrm{C}$; Lanes 7,15 $-64.3^{\circ} \mathrm{C}$; and Lanes $8,16-65^{\circ} \mathrm{C}$. Electrophoresis was run through $1.5 \%$ agarose gels in 1X TBE with VC 100bp Plus DNA Ladder as the MW Marker (M). 
be attributed to the non-specific binding of the primers to the template. On the other hand, lanes 5-8 and 13-16 which correspond to annealing temperatures of $61.1^{\circ} \mathrm{C}$, $63^{\circ} \mathrm{C}, 64.3^{\circ} \mathrm{C}$, and $65^{\circ} \mathrm{C}$ showed faint bands or no bands that indicates the absence of the desired HLA DRB1 amplified product. At high annealing temperatures, the primers are not able to anneal efficiently causing a lower yield of the desired product. $^{14}$ The results for the optimization of annealing temperature indicates that for the PCR reaction, the optimum annealing temperature is $58.8^{\circ} \mathrm{C}$. Patient 1 with annealing temperature set at $58.8^{\circ} \mathrm{C}$ (Lane 4) exhibited 3 distinct bands with one corresponding to around $800 \mathrm{bp}$ which is the expected product length of the HLA DRB1 allele. A similar result is exhibited by Patient 5 (Lane 12) which further affirms that $58.8^{\circ} \mathrm{C}$ as the optimum annealing temperature.

However, extraneous bands and faint bands can also be observed in Lanes 4 and 12 (Figure 3). Non-specific bands are formed when the stringency of PCR conditions is compromised. Non-specific PCR products may have variable lengths and may look like a ladder. When amplifying genomic DNA with highly repetitive sequences which the primers were designed against, smears may be also obtained in the agarose gel. ${ }^{15}$

In order to minimize extraneous bands, a gradient PCR was done using a lowered $\mathrm{MgCl}_{2}$ concentration from $2.5 \mathrm{mM}$ to $1 \mathrm{mM} . \mathrm{Mg}^{2+}$ is an essential cofactor for Taq polymerase to exhibit its function. Lowering the amount of $\mathrm{MgCl}_{2}$ results to a decrease in activity of Taq polymerase which promotes specificity for the desired DNA target. ${ }^{15}$ Figure 4 shows the gel profile for the optimization of $\mathrm{MgCl}_{2}$ concentration run through $1.5 \%$ agarose gels in $1 \mathrm{X} \mathrm{TBE}$ with VC 100bp Plus DNA Ladder as the MW Marker (M).

Lanes 1-8 corresponds to the annealing temperatures $55^{\circ} \mathrm{C}, 55.7^{\circ} \mathrm{C}, 56.9^{\circ} \mathrm{C}, 58.8^{\circ} \mathrm{C}, 61.1^{\circ} \mathrm{C}, 63^{\circ} \mathrm{C}, 64.3^{\circ} \mathrm{C}$, and $65^{\circ} \mathrm{C}$, respectively. In Figure 4 , Lane 4 corresponds to an annealing temperature of $58.8^{\circ} \mathrm{C}$ and shows an ideal band with a high yield but relatively low specificity. However, it is chosen over Lane 5, having an annealing temperature of $61.1^{\circ} \mathrm{C}$, since it is desirable to have a high yield of the DNA for downstream applications. Combining the results obtained from the gel profile of the gradient PCR in Figure 3 and Figure 4, it can be said that a final concentration of $1 \mathrm{mM}$ of $25 \mathrm{mM} \mathrm{MgCl}$ and an annealing temperature of $58.8^{\circ} \mathrm{C}$ are optimum for the final PCR conditions.

\section{Analysis of PCR Products}

Following the optimized PCR conditions, amplification of all ten patient cDNA samples were done to identify the HLA Class II DRB1 allelic variants. The PCR products are shown in Figure 5.

Patients 1, 2, 3, 5, 8, 9, and 10 showed amplified products for HLA Class II DRB1 amplification (Lanes 1, 2, 5, 8, 9, and 10), implying that these samples harbor HLA Class II DRB1. Non-amplification for Patients 4, 6 and 7, on the other

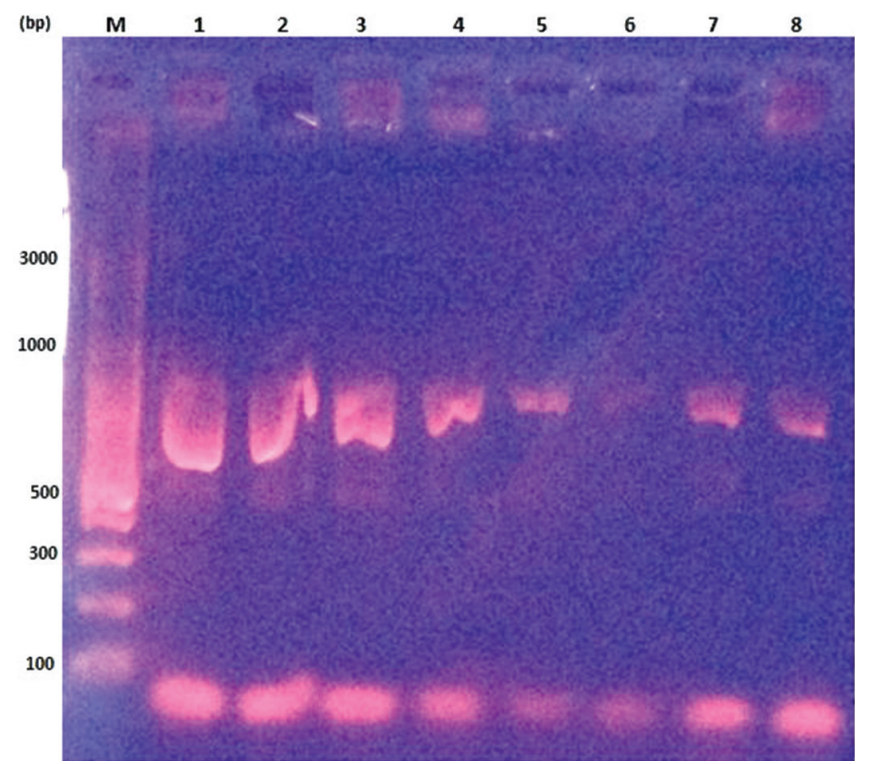

Figure 4. AGE profile of gradient PCR of Patient 1 with 0.4 $\mu \mathrm{L}(1 \mathrm{mM})$ of $25 \mathrm{mM} \mathrm{MgCl}$. Lanes 1-8 correspond to annealing temperatures of $55^{\circ} \mathrm{C}, 55.7^{\circ} \mathrm{C}, 56.9^{\circ} \mathrm{C}$, $58.8^{\circ} \mathrm{C}, 61.1^{\circ} \mathrm{C}, 63^{\circ} \mathrm{C}, 64.3^{\circ} \mathrm{C}$, and $65^{\circ} \mathrm{C}$, respectively. Electrophoresis was run through $1.5 \%$ agarose gels in 1X TBE with VC 100bp Plus DNA Ladder as the MW Marker (M).

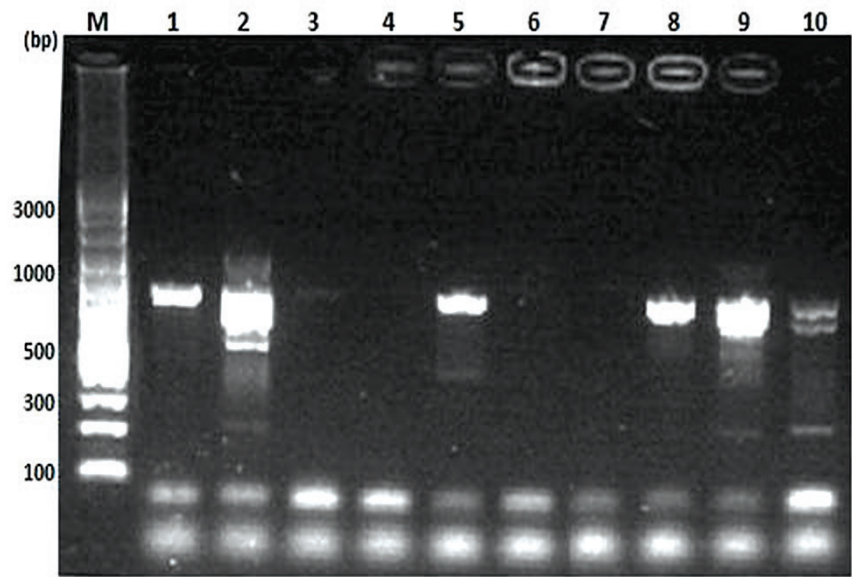

Figure 5. PCR products from running the 10 cancer patient samples. Lanes 1-10 correspond to Patients 1-10, respectively. Electrophoresis was run through 1.5\% agarose gels in 1X TBE with VC 100bp Plus DNA Ladder as the MW Marker (M).

hand, may suggest that the patient expressed a non-DRB1 HLA Class II segment. Further, although all individuals harbor HLA DRB1, nucleotide sequences of HLA alleles may vary up to 57 bases in the peptide-binding region of HLA which may explain the absence of bands for Patients 4,6 , and $7{ }^{16}$ Such molecular differences are important to consider especially in identifying graft-versus-host disease and rejection, and for developing antigenic peptides for dendritic cell cancer vaccines. There are increasing number 
Table 5. Summary of the results obtained from the amplification of the HLA Class II DRB1 in ten Filipino cancer patient samples

\begin{tabular}{|c|c|c|c|}
\hline Sample & $\begin{array}{l}\text { Amplified HLA } \\
\text { Class II DRB1? }\end{array}$ & Allelic Variant with Maximum Score from BLAST & $\begin{array}{l}\text { Gene Accession } \\
\text { Number }\end{array}$ \\
\hline Patient 1 & Yes & $\begin{array}{l}\text { Homo sapiens MHC class II antigen (HLA-DRB1) mRNA, HLA-DRB1*04:06:01 allele, } \\
\text { complete cds }\end{array}$ & MK342635 \\
\hline Patient 2 & Yes & $\begin{array}{l}\text { Homo sapiens MHC class II antigen (HLA-DRB1) mRNA, HLA-DRB1*12:01:01 allele, } \\
\text { complete cds }\end{array}$ & MK342636 \\
\hline Patient 3 & No & N/A & $\mathrm{N} / \mathrm{A}$ \\
\hline Patient 4 & No & N/A & N/A \\
\hline Patient 5 & Yes & $\begin{array}{l}\text { Homo sapiens MHC class II antigen (HLA-DRB1) mRNA, HLA-DRB1*0813 allele, } \\
\text { complete cds }\end{array}$ & MK342637 \\
\hline Patient 6 & No & $\mathrm{N} / \mathrm{A}$ & $\mathrm{N} / \mathrm{A}$ \\
\hline Patient 7 & No & $\mathrm{N} / \mathrm{A}$ & N/A \\
\hline Patient 8 & Yes & $\begin{array}{l}\text { Homo sapiens MHC class II antigen (HLA-DRB1) mRNA, HLA-DRB1*04:05:01 allele, } \\
\text { complete cds }\end{array}$ & MK342638 \\
\hline Patient 9 & Yes & $\begin{array}{l}\text { Homo sapiens MHC class II antigen (HLA-DRB1) mRNA, HLA-DRB1*09:01:02 allele, } \\
\text { complete cds }\end{array}$ & МК342639 \\
\hline Patient 10 & Yes & $\begin{array}{l}\text { Forward: Homo sapiens MHC class II antigen (HLA-DRB1) mRNA, HLA-DRB1*16:02:01 } \\
\text { allele, complete cds }\end{array}$ & МK342640 \\
\hline
\end{tabular}

${ }^{b}$ Consensus sequences for patients 1, 2, 5, 8, 9, and 10 are shown in Table C1 (Appendix C).

of evidences that point toward diminishing survival among patients subjected to hematopoietic stem cell transplantation due to changes, even if very minimal, in HLA alleles between donor and recipient. ${ }^{17}$ Moreover, peptide binding is also dependent in the HLA allele that is present in an individual and this is a critical factor in designing antigenic peptides for dendritic cell cancer vaccine as it will only work on individuals who harbor the specific HLA allele. ${ }^{18}$ The other small faint bands that were visible besides the desired PCR product may be due to partial amplification of the desired product or it may be the primer itself. It is important to note that other factors may potentially affect the success of implementing this assay which may come from the source as in harvest of hematopoietic stem cells via leukapheresis. ${ }^{19}$ To confirm the identity of the amplified products, they were gel-purified and sent for sequencing. However, Patient 3 (Lane 3) does not meet the requirement for DNA sequencing because of its low concentration as seen from the faint band at around $800 \mathrm{bp}$.

\section{DNA Sequencing Results}

Out of the 10 patients analyzed, 6 showed positive amplification of HLA DRB1. Table 5 presents the summary of the results obtained from the amplification of HLA Class II DRB1 of the ten Filipino cancer patient samples. The sequencing results show that the HLA DRB1 allelic variants can be amplified using the primers that were designed. However, it was also seen that it can be crossreactive to other HLA DRB allelic variants as shown by Patient 2 who had higher max score and \% identity for HLA DRB3 due to high sequence similarity between DRB1 and DRB3. Nevertheless, this study was able to suggest the DRB allelic variants contained in the patient samples. Thus, the samples can be utilized for the downstream development of antigenic peptides for dendritic cell cancer vaccine.

\section{CONCLUSION}

From this study, primers against the HLA Class II DRB1 were successfully designed allowing the amplification of HLA Class II DRB1 allelic variants from cancer patient samples of unstimulated stem cell with high specificity and minimal cross-reactivity. PCR conditions were successfully optimized in terms of $\mathrm{MgCl}_{2}$ concentration at $1 \mathrm{mM}$ and annealing temperature of $58.8^{\circ} \mathrm{C}$.

Since the success of an immunotherapy via dendritic cell vaccine relies mainly on the HLA type of an individual, it is recommended to test the primers that were designed with other samples containing known HLA alleles apart from HLA DRB1. If it is found that the primers are specific to HLA DRB1 then it can be used as a primer for standard HLA typing methods. Moreover, the primers can be used in multiplex PCR for HLA DRB1 identification in individuals. More patient samples can also be used for identifying HLA allelic variants to further validate the primers designed. It is also recommended that the patients that were tested positive for HLA Class II DRB1 be utilized for downstream applications such as the development of antigenic peptides for cancer immunotherapy treatment such as dendritic cell vaccine.

\section{Statement of Authorship}

All authors participated in the data collection and analysis, and approved the final version submitted.

\section{Author Disclosure}

All authors declared no conflicts of interest.

\section{Funding Source}

This research was supported in part by the National Institutes of Health - University of the Philippines Manila, 
UP Manila College of Medicine, and Lung Center of the Philippines.

\section{Supplemental data}

Supplemental data (Sequencing results for the ten cancer patient samples using primers designed for HLA Class II DRB1*1502) are available upon request to the corresponding author.

\section{REFERENCES}

1. Ayala García MA, González Yebra B, López Flores AL, Guaní Guerra E. The major histocompatibility complex in transplantation. J Transplant. 2012; 2012:842141. doi: 10.1155/2012/842141.

2. Neefjes J, Jongsma MLM, Paul P, Bakke O. Towards a systems understanding of $\mathrm{MHC}$ class I and $\mathrm{MHC}$ class II antigen presentation. Nat Rev Immunol. 2011 Nov; 11(12):823-36. doi: 10.1038/nri3084.

3. Jorgensen KW, Buus, S, Nielsen M. Structural properties of MHC class II ligands, implications for the prediction of MHC class II epitopes. PLoS One. 2010 Dec; 5(12):e15877. doi:10.1371/journal. pone. 0015877

4. Mahdi BM. A glow of HLA typing in organ transplantation. Clin Transl Med. 2013 Feb; 2(1):6. doi.10.1186/2001-1326-2-6

5. Immuno Polymorphism Database [Internet]. 2006 [cited 2017 May]. Available from: https://www.ebi.ac.uk

6. Bugawan TL, Chang JD, Klitz W, Erlich HA. PCR/oligonucleotide probe typing of HLA class II alleles in a Filipino population reveals an unusual distribution of HLA haplotypes. Am J Hum Genet. 1994 Feb; 54(2):331-40.

7. Howell WM, Carter V, Clark B. The HLA system: immunobiology, HLA typing, antibody screening and crossmatching techniques. J Clin Pathol. 2010 May; 63(5):387-90. doi: 10.1136/jcp.2009.072371.

8. Stern LJ, Calvo-Calle JM. HLA-DR: molecular insights and vaccine design. Curr Pharm Des. 2009; 15(28):3249-61.
9. Chuang LY, Cheng YH, Yang CH. Specific primer design for the polymerase chain reaction. Biotechnol Lett. 2013 Oct; 35(10):1541-9. doi: 10.1007/s10529-013-1249-8.

10. Buzdin A, Lukyanov S. Nucleic acids hybridization: modern applications. Netherlands: Springer; 2007. p. 112.

11. Levesque-Sergerie JP, Duquette M, Thibault C, Delbecchi L, Bissonnette N. Detection limits of several commercial reverse transcriptase enzymes: impact on the low- and high-abundance transcript levels assessed by quantitative RT-PCR. BMC Mol Biol. 2007 Oct; 8:93.

12. Sipos R, Szekely AJ, Palatinszky M, Revesz S, Marialigeti K, Nikolausz $\mathrm{M}$. Effect of primer mismatch, annealing temperature and PCR cycle number on $16 \mathrm{~S}$ rRNA gene-targeting bacterial community analysis. FEMS Microbiol Ecol. 2007 May;60(2):341-50.

13. Ruiz-Villalba A, van Pelt-Verkuil E, Gunst QD, Ruijter JM, van den Hoff MJB. Amplification of nonspecific products in quantitative polymerase chain reactions (qPCR). Biomol Detect Quantif. 2017 Nov; 14:7-18. doi: 10.1016/j.bdq.2017.10.001.

14. Verma K, Dalal J, Sharma S. Scientific concepts of polymerase chain reaction (PCR). Int J Pharm Sci Res. 2014 Aug; 5(8):3086-95.

15. Lorenz TC. Polymerase chain reaction: basic protocol plus troubleshooting and optimization strategies. J Vis Exp. 2012 May; (63):e3998. doi: 10.3791/3998..

16. Buhler S, Sanchez-Mazas A. HLA DNA sequence variation among human populations: molecular signatures of demographic and selective events. PLoS One. 2011 Feb; 6(2):e14643. doi: 10.1371/journal. pone. 0014643 .

17. Wiebe C, Pochinco D, Blydt-Hansen TD, Ho J, Birk PE, Karpinski $\mathrm{M}$, et al. Class II HLA epitope matching -- a strategy to minimize de novo donor-specific antibody development and improve outcomes. Am J Transplant. 2013 Dec;13(12):3114-22. doi: 10.1111/ajt.12478. Epub 2013 Oct 25.

18. Cintolo JA, Datta J, Matthew SJ, Czerniecki BJ. Dendritic cellbased vaccines: barriers and opportunities. Future Oncol. 2012 Oct; 8(10):1273-99. doi: 10.2217/fon.12.125.

19. Potapov V, Ong JL. Examining sources of error in PCR by singlemolecule sequencing. PLoS One. 2017 Jan; 12(1):e0169774. doi: 10.1371/journal.pone.0169774.

\section{APPENDICES}

Appendix A. HLA Class II DRB1*1502 DNA sequence used for primer design. The sequence was derived from IPD-IMGT/ HLA Accession No. HLA00867

Nucleotide Sequence Data (801bps)

ATGGTGTGTCTGAAGCTCCCTGGAGGCTCCTGCATGACAGCGCTGACAGTGACACTGATGGTGCTGAGCTCCCCACTGGCTTTGTCTGGGGA CACCCGACCACGTTTCCTGTGGCAGCCTAAGAGGGAGTGTCATTTCTTCAATGGGACGGAGCGGGTGCGGTTCCTGGACAGATACTTCTATAAC CAGGAGGAGTCCGTGCGCTTCGACAGCGACGTGGGGGAGTTCCGGGCGGTGACGGAGCTGGGGCGGCCTGACGCTGAGTACTGGAACAGC CAGAAGGACATCCTGGAGCAGGCGCGGGCCGCGGTGGACACCTACTGCAGACACAACTACGGGGTTGGTGAGAGCTTCACAGTGCAGCGGC GAGTCCAACCTAAGGTGACTGTATATCCTTCAAAGACCCAGCCCCTGCAGCACCACAACCTCCTGGTCTGCTCTGTGAGTGGTTTCTATCCAG GCAGCATTGAAGTCAGGTGGTTCCTGAACGGCCAGGAAGAGAAGGCTGGGATGGTGTCCACAGGCCTGATCCAGAATGGAGACTGGACCTTC CAGACCCTGGTGATGCTGGAAACAGTTCCTCGAAGTGGAGAGGTTTACACCTGCCAAGTGGAGCACCCAAGCGTGACAAGCCCTCTCAAGTG GAATGGAGAGCACGGTCTGAATCTGCACAGAGCAAGATGCTGAGTGGAGTCGGGGGCTTTGTGCTGGGCCTGCTCTTCCTTGGGGCCGGGCT GTTCATCTACTTCAGGAATCAGAAAGGACACTCTGGACTTCAGCCAACAGGATTCCTGAGCTG 
Identification of HLA Class II DRB1 Allelic Variant in Filipino Cancer Patient Samples

Appendix B. RNA concentrations of the ten cancer patient samples measured using QuantusFluorometer

\begin{tabular}{crrrr} 
& \multicolumn{3}{c}{ RNA $(\mathbf{n g} / \mu \mathrm{L})$} & Average Concentration $(\mathrm{ng} / \mu \mathrm{L})$ \\
\cline { 2 - 4 } Sample & Trial 1 & Trial 2 & Trial 3 & 79.3 \\
Patient 1 & 79.6 & 79.2 & 79.0 & 95.2 \\
Patient 2 & 95.6 & 95.2 & 94.8 & 18.0 \\
Patient 3 & 18.0 & 18.0 & 18.0 & 27.5 \\
Patient 4 & 27.6 & 27.5 & 27.4 & 39.0 \\
Patient 5 & 39.2 & 39.0 & 38.8 & 105.0 \\
Patient 6 & 105.0 & 105.0 & 105.0 & 33.3 \\
Patient 7 & 33.3 & 33.3 & 33.2 & 53.1 \\
Patient 8 & 53.6 & 53.0 & 52.7 & 68.0 \\
Patient 9 & 68.2 & 68.0 & 67.9 & 54.2 \\
Patient 10 & 54.5 & 54.3 & 53.7 & \\
\hline
\end{tabular}

Appendix C. Consensus sequences from the forward and reverse sequences aligned using MEGA7

\begin{tabular}{ll}
\hline Patient 1 & \multicolumn{1}{c}{ Consensus Sequence } \\
(Gene Accession & TGGCAGCTTTGACAGTGACACTGATGGTGCTGAGCTCCCCACTGGCTTTGGCTGGGGACACCCGAC \\
number: MK342635) & GACAGATACTTCCATAACCAGGAGGAGTACGTGCGCTTCGACAGCGACGTGGGGGAGTACCGGGCG \\
& GTGACGGAGCTGGGGCGGCTGATGCCGAGACTGGAACAGCCAGAAGGACCTCCTGGAGCAGAGGC \\
& GGGCCCAGGTGGACACTTACTGCAGACACAACTACGGGGTTGTGGAGAGCTTCACAGTGCAGCGGC \\
& GAGTCCATCCTGAGGTGACTGTGTATCCTGCAAAGACCCAGCCCCTGCAGCACCACAACCTCCTGGTCT \\
& GCTCTGTGAGTGGTTTCTATCCAGGCAGCATTGAAGTCAGGTGGTTCCGGAACGGCCAGGAAGAGA \\
& AGACTG
\end{tabular}

Patient 2 TCTGACAGTGACACTGATGGTGCTGAGCTCCCGACTGGCTTTCGCTGGGGACACCCGACCACGTTTCTTG (Gene Accession GAGTTGTCTAAGTCTGAGTGTCATTTCTTCAATGGGACGGAGCGGGTGCGGTTCCTGGAGAGA number: MK342636) CACTTCCATAACCAGGAGGAGTTCCTGCGCTTCGACAGCGACGTGGGGGAGTTCCGGGCGGTGACG GAGCTGGGGCGGCCTGTCGCCGAGTCCTGGAACAGCCAGAAGGACCTCCTGGAACACAAGCGCGCCCC GGTGGACACTTATTCAGACACAACTACGGGGTTGTGGAGAGCTTCACAGTGCAGCGGCGAGTCCATCCT CAGGTGACTGTGTATCCTTCAAAGACCCAGCCCCTGCAGCACCACAACCTCCTGGTCTGTTCTGTGAGTG GTTTCTATCCAGGCAGCATTGAAGTCAGGTGGTTCC

Patient 5

(Gene Accession number: MK342637)

ATAACCAAGAGGAGTACGCGCGCTTCGACAGCGACGTGGGGGAGTACCGGGCGGT GAGGGAGCTGGGGCGGCCTGATGCCGAGTACTGGAACAGCCAGAAGGACCTCCTGGAACACAAGC GGGGCCAGGTGGACACTTACTGCAGACACAACTACGGGGTTGGTGAGAGCTTCACAGTGCAGCGGC GAGTCCATCCTCAGGTGACTGTGTATCCTGCAAAGACCCAGCCCCTGCAGCACCACAACCTCCTGGTCT GTTCTGTGAGTGGTTTCTATCCAGGCAGCATTGAAGTCAGGTGGTTCCGGAACGGCCAGGAAGAGA AGGCTGGGGTGGTGTCCACAGGCCTGATCCACAATGGAGACTGGACCTTCCAGACCCTGGTGATGCTG GAAACAGTTCCTCGGAGTGGAGAGGTTTACACCTGCCAAGTGGAGCACCCAAGCGTAACGAGCCCTCT CACAGTGGAATGGAGTGCACGGTCTGAATCTGCACAGAGCAAGATGCTGAGTGGAGTCGGGGGCTTT GTGGTGGGCCTGCTCTTCCTTGGGGCCGGGCTGTTCATTTACTTCAAGAT

Patient 8

(Gene Accession number: MK342638)

GGAGCAGAGGCGGGCCGCGGTGGACACCTACTGCAGACACAACTACGGGGTTGGTGAGAGCTTCA CAGTGCAGCGGCGAGTCCATCCTGAGGTGACTGTGTATCCTGCAAAGACCCAGCCCCTGCAGCACCA CAACCTCCTGGTCTGCTCTGTGAGTGGTTTCTATCCAGGCAGCATTGAAGTCAGGTGGTTCCGGAACGGC CAGGAAGAGAAGACTGGGGTGGTGTCCACAGGCCTGATCCAGAATGGAGACTGGACCTTCCAGACCCTG GTGATGCTGGAAACAGTTCCTCGGAGTGGAGAGGTTTACACCTGCCAAGTGGAGCACCCAAGCGTGAC GAGCCCTCTCACAGTGGAATGGAGAGCACGGTCTGAATCTGCACAGAGCAAGATGCTGAGTGGAGTC GGGGGCTTTGTGCTGGGCCTGCTCTTCCTTGGGGCCGGGCTGTTCATCTACTTCAGGAATCAGAAA

Patient 9

(Gene Accession number: MK342639)

AGGTGGACACCGCGTGCAGACACAACTACGGGGTTGGTGAGAGCTTCACAGTGCAGAGGCGAGTC CATCCTGAGGTGACTGTGTATCCTGCCAAGACTCAGCCCCTGCAGCACCACAACCTCCTGGTCT GCTCTGTGAGTGGTTTCTATCCAGGCAGCATTGAAGTCAGGTGGTTCCGGAACGGCCAGGAAGAGA AGGCTGGGGTGGTGTCCACAGGCCTGATCCAGAATGGAGACTGGACCTTCCAGACCCTGGTGATGCTG GAAACAGTTCCTCGGAGTGGAGAAGTTTACACCTGCCAAGTGGAGCACCCAAGTGTGATGAGCCCTCTCA CAGTGGAATGGAGAGCACGGTCTGAATCTGCACAGAGCAAGATGCTGAGTGGAGTCGGGGGCTTTTGT GCTGGGCCTGCTCTTCCTTGGGGCCGGGCTGTTCATCTACTTCAAGAATCAGAAAGGACAC

Patient 10

(Gene Accession number: MK342640)
TTTCCTGTGGCAGCCTAAGAGGGAGTGTCATTTCTTCAATGGGACGAAGCGGGTGCGGTTCCTGGA CAGATACTTCTATAACCACGAAGAGTCCGTGCGCTTCAACAGCGACTTGGGGGAATACCGGGCGGT GACGGAGCTGGGGCGGCCTGACGCTGAATACTGGAACAGCCACAAGGACCTCCTGGAAGATAGGC GCGCCGCGGTGGACACCTACTGCAGACACAACTACGGGGTTGGTGAGAGCTTCACAGTGCAGCGGC GAGTCCAACCTAAGGTGACTGTATATCCTTCAAAGACCCAGCCCCTGCAGCACCACAACCTCCTGGTCT GCTCTGTGAGTGGTTTCTATCCAGGCAGCATTGAAGTCAGGTGGTTCCTGAACGGCCAGGAAGAGA AGGCTGGGATGGTGTCCACAGGCCTGATCCAGAATGGAGACTGGACCTTCCAGACCCTGGTGATGCTG GAAACAGTTCCTCGAAGTGGAGAGGTTTACACCTGCCAAGTGGAGCACCCAAGCGTGACAAGCCCTCT CACAGTGGAATGGAGACCCTCGTCAGTTTCTGCACAGAGCTTCATGATGAGAGGAGTCGGGGGGCTC 